\title{
Tubercular Papillitis of Vater
}

\author{
Tateki Yamane $^{1 *}$, Takayuki Ishii ${ }^{2}$, Akira Umeda $^{3}$, Shigeharu Takagi ${ }^{4}$, Hitoshi Shimao ${ }^{5}$ \\ ${ }^{1}$ Division of Gastroenterology, Department of Internal Medicine, The International University of Health and Welfare, Shioya Hospi- \\ tal, Yaita, Japan; ${ }^{2}$ Sanikukai Family Clinic, Funabashi, Japan; ${ }^{3}$ Division of Pulmonology, Department of Internal Medicine, The In- \\ ternational University of Health and Welfare, Shioya Hospital, Yaita, Japan; ${ }^{4}$ Division of Neurology, Department of Internal Medi- \\ cine, The International University of Health and Welfare, Shioya Hospital, Yaita, Japan; ${ }^{5}$ Department of Surgery, The International \\ University of Health and Welfare, Shioya Hospital, Yaita, Japan. \\ Email: *yamane@iuhw.ac.jp
}

Received August 14 $4^{\text {th }}, 2012$; revised September 20 ${ }^{\text {th }}, 2012$; accepted October $25^{\text {th }}, 2012$

\begin{abstract}
A middle-aged man with no symptom or history visited our hospital due to duodenal abnormality and fecal occult blood detected by health screening. Upper gastrointestinal endoscopy showed marked enlargement of the papilla of Vater surrounded by nodules, and biopsy disclosed non-caseating granuloma. Colonoscopy yielded findings suggestive of intestinal tuberculosis such as annular ulcers. Examination of the biopsy samples failed to make a diagnosis of tuberculosis, and no pulmonary lesion was found, but whole-blood interferon gamma release assay was positive. The duodenal lesion was also suspected to be tubercular, so antitubercular drugs were administered. As this resulted in the resolution of both lesions, a definite diagnosis of tubercular papillitis of Vater was made. Duodenal tuberculosis is rare, and a localized lesion of the major duodenal papilla is extremely rare. However, this case suggests that tuberculosis must be considered in the differential diagnosis of lesions of the papilla of Vater.
\end{abstract}

Keywords: Duodenal Tuberticulosis; Papillitis of Vater; Intestinal Tuberculosis

\section{Introduction}

Intestinal tuberculosis may occur in any part of the digestive tract. However, duodenal tuberculosis is rare because of fewness of lymph follicles in the luminal wall, influence of gastric acid, and rapid passage of intestinal contents, etc. In the duodenal tuberculosis, a localized lesion of the major duodenal papilla is extremely rare. We encountered a case of tubercular papillitis of Vater, so report it here.

\section{Case Report}

A 47-year-old male visited our hospital for close evaluation due to enlargement of the papilla of Vater suggested by upper gastrointestinal barium radiography and fecal occult blood detected on mass health screening. He had no symptom or history of major diseases. He had no history of smoking and drank only occasionally. He was a company employee, and none of his colleagues or family had tuberculosis. There had been no episode of overworking or lack of sleep.

There was no abnormality of the physical condition including the nutritional state.

Blood tests showed no abnormality of the cell counts or biochemical items, and a slight inflammatory reaction

\footnotetext{
${ }^{*}$ Corresponding author.
}

with a CRP of $1.1 \mathrm{mg} / \mathrm{dL}$ was noted. Upper and lower gastrointestinal endoscopy was performed.

Upper gastrointestinal endoscopic findings: While no abnormality was noted in the esophagus, stomach, or duodenal bulb, the major duodenal papilla was enlarged markedly and was covered by thin fur, and multiple nodules were noted around it (Figures 1(a) and (b)). No abnormality was observed in other areas of the descending part of the duodenum.

Histopathological findings: Biopsy revealed non-caseating granuloma in the lamina propria mucosae (Figure 2(a)). Findings on Ziehl-Neelsen (ZN) staining were negative.

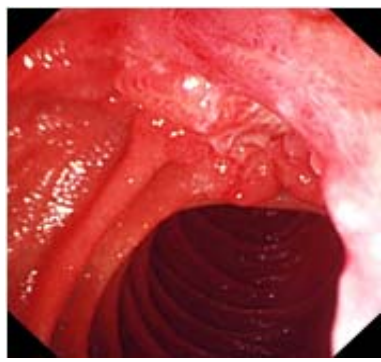

(a)

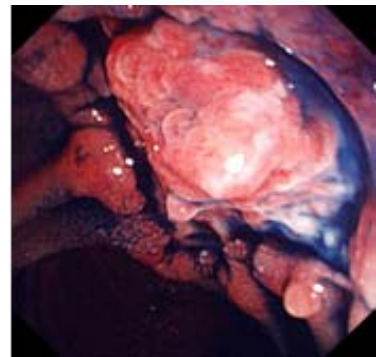

(b)
Figure 1. Upper gastrointestinal endoscopic findings. The major duodenal papilla was enlarged, and multiple nodules were noted around it. (a) Conventional view; (b) Indigo carmine spraying view. 
On MRCP, no abnormality was noted in the bile or pancreatic duct (Figure 2(b)). Abdominal CT or ultrasonography (US) also showed no abnormality.

Colonoscopic findings: While no abnormality was observed from the rectum to transverse colon, annular ulcers were noted in the ascending colon sporadically, there were scarred areas with discoloration in the cecum, and Bauhin's valve was disfigured and loosened (Figures $\mathbf{3 ( a )}$ and (b)).

The colon lesion was morphologically considered to

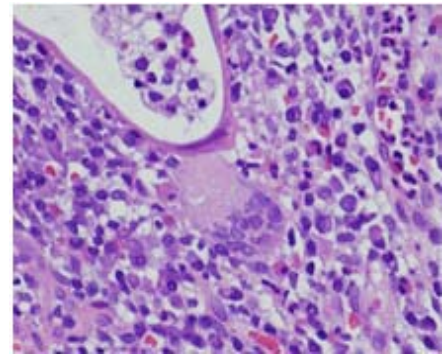

(a)

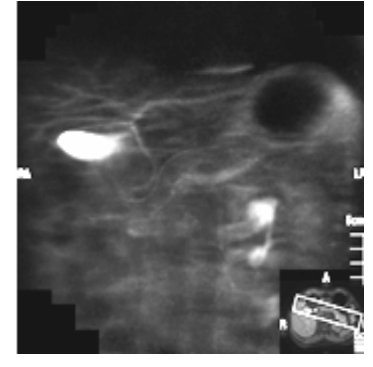

(b)
Figure 2. (a) Histopathological findings in a biopsy specimen (HE staining). Non-caseating granuloma was found in the lamina propria mucosae; (b) MRCP finding. No abnormality was noted.

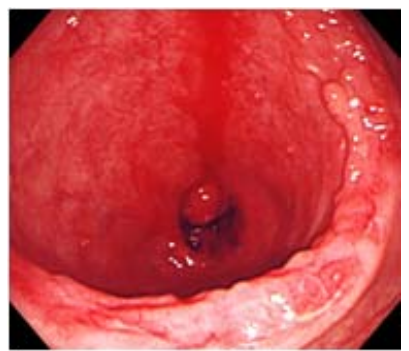

(a)

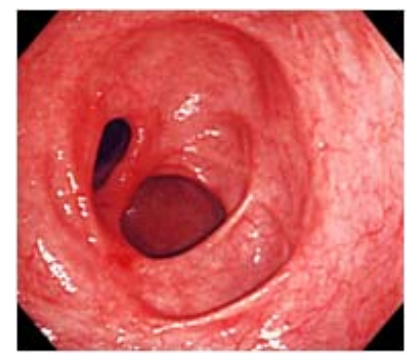

(b)
Figure 3. Colonoscopic findings. (a) Annular ulcers were found in the ascending colon; (b) Scarred areas was noted in the cecum, and Bauhin's valve was disfigured. be tuberculosis, but biopsy yielded no decisive finding such as granuloma, and the results of ZN staining were negative. The PCR test for tubercle bacillus and a tubercle bacillus culturing test using biopsy samples were both negative, but whole-blood interferon gamma release assay (IGRA) was positive. Chest CT showed no abnormality in the lung field, and no gross lesion was found by small intestinal barium radiography. HIV was negative. While the duodenal lesion was non-caseating, it was suspected to be tubercular as granuloma was detected. Antitubercular therapy using 4 drugs was carried out. After administering isoniazid (INH) at $0.4 \mathrm{~g} /$ day, refampicin (RFP) at $0.45 \mathrm{~g} /$ day, ethambutol at $0.75 \mathrm{~g} /$ day, and pyrazinamide at $1.5 \mathrm{~g} /$ day for 2 months, the administration of INH and RFP was continued at the same doses over 4 months. This regimen is one of standard drug therapy of tuberculosis in Japan. Adverse effect was not found during the therapy. Endoscopy was performed again immediately after the 6-month antitubercular therapy.

Colonoscopic findings: The annular ulcers in the ascending colon were scarred (Figure 4(a)).

Upper gastrointestinal endoscopic findings: Enlargement of the major duodenal papilla was resolved, no fur was observed, and the surrounding nodules had disappeared (Figures 4(b) and (c)).

From these findings, the duodenal as well as colon lesion was diagnosed to be tubercular, and both lesions were considered to have been cured.

\section{Discussion}

The lesion of the major duodenal papilla in this patient could not be diagnosed as tubercular by macroscopic examination alone. By biopsy, however, granuloma was detected, although it was non-caseating, and colonoscopy revealed changes characteristic of intestinal tuberculosis, allowing us to suspect the duodenal and colon lesions to be a series of tubercular changes. After the administration of antitubercular drugs, cure of the duodenal as well

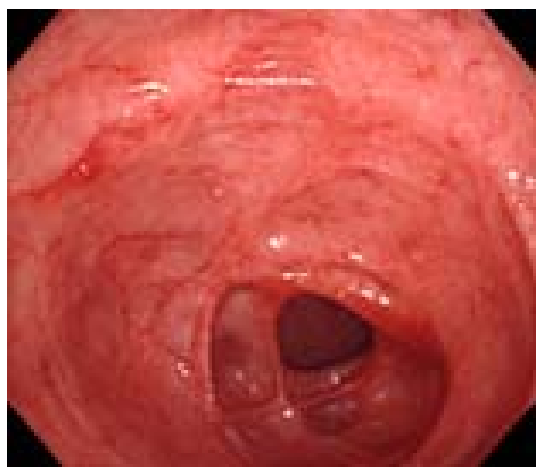

(a)

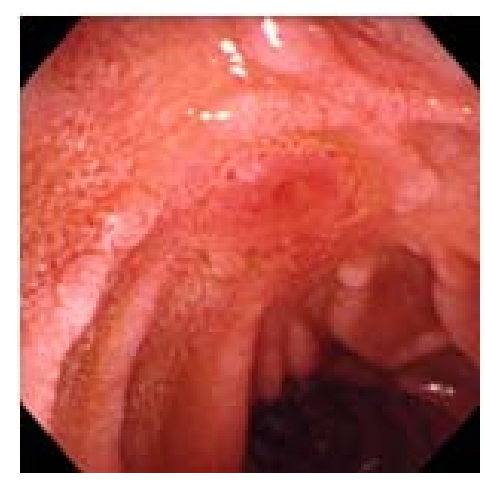

(b)

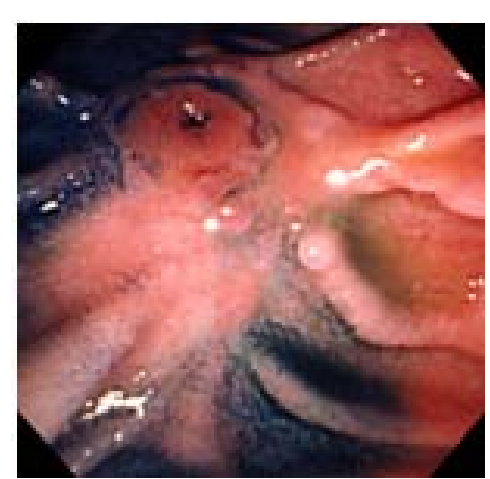

(c)

Figure 4. Endoscopic findings after the antitubercular therapy. (a) The annular ulcers in the ascending colon were scarred; (b), (c): Enlargement of the major duodenal papilla was resolved, and the surrounding nodules had disappeared; ((b) Conventional view; (c) Indigo carmine spraying view). 
as colon lesion was confirmed. Paustian et al. proposed that at least one of the following is necessary for a definitive diagnosis of intestinal tuberculosis: 1) Detection of tubercle bacillus by culturing of the intestinal wall, mesentery, or regional lymphatic tissue; 2) Detection of caseating granuloma at the lesion; 3) Demonstration of tuberculosis by pathological examination of the lesion; and 4) pathological demonstration of tuberculosis in the resected mesenteric lymph nodes and gross confirmation of typical tubercular changes during surgery [1]. It has been reported that the accuracy of the histological or bacteriological diagnosis of intestinal tuberculosis using biopsy samples obtained by endoscopic guidance is not necessarily high. In Japan, the detection rate of caseating granuloma in biopsy samples has been reported to be $0 \%$ $40 \%$, the positive rate on acid-fast staining to be $0 \%$ $36 \%$, positive rate of tubercle bacillus by the PCR test to be $30 \%-64.1 \%$, and positive rate of cultures for tubercle bacillus to be $6 \%-54.5 \%$. In our patient, also, the results of these tests were all negative. According to the criteria of Paustian et al., the digestive tract lesion in our patient should not be diagnosed as tuberculosis, but some investigators propose to diagnose tuberculosis according to the response to antitubercular medication, and a diagnosis of intestinal tuberculosis was made in our patient in consideration of the treatment results. In our patient, a positive IGRA result also contributed to the diagnosis. This test is expected to be useful as a substitute for the tuberculin reaction as its results are not affected by past BCG inoculation or most non-tubercular mycobacterial infections, and as its sensitivity and specificity for the diagnosis of tuberculosis have been reported at $89 \%$ and $98 \%$, respectively [2]. Its usefulness for the diagnosis of intestinal tuberculosis has not been established, and further evaluation by the accumulation of cases is anticipated.

In Japan, the rate of decrease in tuberculosis slowed with the aging of the population and the increase in immunological diseases, and its incidence has been increasing since 1996. The incidence of intestinal tuberculosis is also expected to increase, and it remains a disease that must be remembered in the differential diagnosis of inflammatory diseases of the digestive tract. Tuberculosis may occur in any part of the digestive tract, but it does not frequently affect the duodenum. In Japan, duodenal tuberculosis accounts for only $2.5 \%-5.4 \%$ of all intestinal tuberculosis, being the rarest following gastric tuberculosis [3]. Tuberculosis affects the stomach or duodenum infrequently, presumably because lymph follicles in the luminal wall are few, the growth of the bacillus is inhibited by gastric acid, bacillus survival is difficult because of the rapid passage of intestinal contents, antibacterial and mucosa-protecting effects are provided by rich mucus secretion, etc. As a result of a search of the Japana Centra Revuo Medicina and MEDLINE between
1951 and 2008, 49 cases of duodenal tuberculosis were found. The patients were relatively young, with a mean age of 45.6 years, partly because elderly people become less susceptible to tuberculosis due to the atrophy of lymph follicles in the duodenal wall. Our patient was 47 years old, being close to the average age of the patients in the literature. In the 49 patients, tuberculosis occurred most frequently in the descending part, followed by the ascending part, horizontal part, and bulb in this order. However, none of these cases had a lesion in the major duodenal papilla, making our patient a rare case. The gross findings in the 49 patients were primarily stenosis and ulcer, and abdominal pain or abdominal fullness was noted in most patients. However, our patient showed no stenosis or deep ulcer and was asymptomatic. Duodenal tuberculosis was complicated by pulmonary tuberculosis in 26 , but by lower intestinal tuberculosis in only 18 , of the 49 patients. In our patient, there was no pulmonary lesion, and the detection of a colon lesion led to the diagnosis. Caution is necessary in diagnosing duodenal tuberculosis as it is often unaccompanied by other lesions in the digestive tract.

Intestinal tuberculosis is considered to be transmitted primarily by 2 routes: Hematogenous or lymphogenous infection of the intestinal wall from pulmonary lesions of tuberculosis, and direct infection of the intestinal tract due to swallowing of tubercle bacillus. In our patient, no pulmonary tuberculosis was noted, and abdominal CT or US showed no enlargement of lymph nodes around the intestine. Therefore, hematogenous or lymphogenous infection is considered unlikely, and the disease is speculated to have been caused by direct infection due to swallowing of tubercle bacillus. Swallowed tubercle bacillus is considered to have attached to the mucosa near the major duodenal papilla, entered the subcumosa, proliferated in lymph follicles, even though they are few in this region, and formed a lesion. Extension of inflammation from the gallbladder or bile duct to the major duodenal papilla has been reported as a rare pattern of the development of duodenal tuberculosis [4], but this possibility was excluded in our patient by imaging studies.

The gross morphology of duodenal tuberculosis was classified into ulcerative, hyperplastic, infiltrative, and peritoneal types by Balikian et al. [5]. Also, multiple small elevations in the margins of ulcer lesions have been reported as a characteristic endoscopic finding of ulcerative-type duodenal tuberculosis. In our patient, the disease is considered to have been the ulcerative type, because the lesion was covered by fur, and the duodenal papilla was surrounded by multiple small elevations, being in agreement with the reported characteristic endoscopic findings. Retrospectively, we should have interpreted these changes as gross signs of tuberculosis. Tuberculosis, while rare, should be considered in the differential 
diagnosis of lesions of the papilla of Vater.

\section{REFERENCES}

[1] F. F. Paustian and G. L. Monto, "Tuberculosis of the Intestines,” In: H. L. Bockus, Ed., Gastroenterol, Vol. 2, 1976, pp. 750-777.

[2] T. Mori, M. Sakatani, F. Yamagishi, T. Takashima, Y. Kawabe, et al., "Specific Detection of Tuberculosis Infection with an Interferon- $\gamma$-Based Assay Using New Antigens," American Journal of Respiratory and Critical Care Medicine, Vol. 170, No. 1, 2004, pp. 59-64.
doi:10.1164/rccm.200402-1790C

[3] T. Yao, T. Sakurai, J. Yamamoto, I. Hirai, H. Furukawa, et al., "Intestinal Tuberculosis in Recent Years-Analysis of the Reported Cases during the Last Decade in Japan," Stomach and Intestine, Vol. 30, No. 4, 1995, pp. 485-490.

[4] E. D. Palmer, "Tuberculosis of the Stomach and the Stomach in Tuberculosis," American Review of Tuberculosis, Vol. 61, No. 1, 1950, pp. 116-130.

[5] J. P. Balikian, S. M. Yenikomshian and Y. D. Jidejian, "Tuberculosis of the Pyloro-Duodenal Area," American Journal of Roentgenology, Vol. 101, No. 2, 1967, pp. 414-420. 